\title{
Dantzig-Wolfe decomposition for linearly constrained stable set problem
}

\author{
Virginie Gabrel
}

\begin{abstract}
Résumé
Nous considérons l'application d'un schéma de décomposition de Dantzig-Wolfe sur un programme linéaire en variables 0 - 1 dans lequel un sous-ensemble de contraintes definit un polytope de stable. Nous comparons les relaxations linéaires du programme de départ et du programme maître (obtenu en décomposant sur les contraintes de stable) en fonction de différentes représentations du polytope de stable. Dans le cas de graphe parfait (et en particulier de graphe de co-comparabilité), la relaxation linéaire du programme maître peut être résolue en un temps polynomial alors que ce n'est pas le cas dans le cas général. En conséquence, il peut être intéressant de décomposer uniquement sur un sous-ensemble de contraintes de stable (celles définissant un problème de stable sur un graphe parfait) de façon à définir un nouveau programme maître pouvant être résolu en un temps polynomial et, renforcant la relaxation continue du programme de départ.
\end{abstract}

Mots-clefs : Decomposition de Dantzig-Wolfe, polytope du stable, graphe parfait

\begin{abstract}
We consider the Dantzig-Wolfe decomposition for 0-1 linear programming when a subset of constraints defines a stable set polytope. We compare linear relaxations of both initial program and master program (obtained by decomposing on stable set constraints) with regards to various stable set polytope representations. For perfect graphs (in particular for cocomparability graph), the linear relaxation of the master program is easy to solve while for general graphs, its optimal value cannot be computed in polynomial time. Consequently, we propose to decompose only on a subset of the stable set constraints (those associated with "polynomial" stable set problems) in order to define another master program for which the LP-relaxation is easy to solve and remains stronger than the classical LP-relaxation of the initial program.
\end{abstract}

Key words : Dantzif-Wolfe decomposition, stable set polytope, perfect graph

*LAMSADE, Université Paris-Dauphine, place du Maréchal de Lattre de Tassigny, 75775 Paris cedex 16, France. gabrel@lamsade.dauphine.fr 


\section{Introduction}

Large-scale 0-1 linear programs often present a strong structure on which a decomposition scheme can be applied. One of the most famous decomposition scheme in linear programming is the Dantzig-Wolfe decomposition proposed in [5]. This decomposition scheme may be applied to 0-1 linear programs [2]. It leads to reformulate the initial program into another one, called the master program, only containing a sub-set of constraints on some original variables plus additional variables representing all the $0-1$ solutions of the decomposed constraints. When the polytope defined by the decomposed constraints does not present the integrality property, it is well-known that the LP-relaxation of the master program is a strengthened formulation of the initial program continuous relaxation. But, in this case, the LP-relaxation of the master program is difficult to solve since the variables involved in an optimal solution which represent some $0-1$ solutions of the decomposed constraints, cannot be determined in polynomial time (the problem of determining a particular 0-1 solution of the decomposed constraints is called the auxiliary problem).

In this paper, we focus on a particular class of 0-1 linear programs in which a sub-set of constraints defines a stable set polytope. We study the difficulty and the interest of applying the Dantzig-Wolfe decomposition scheme on the stable set constraints. When the decomposed constraints represents a stable set problem in a perfect graph, the auxiliary problem can be solved in polynomial time and the optimal solution value of the master program LP-relaxation can be obtained by column generation procedure. For some particular classes of perfect graphs (like interval or cocomparability graphs), another formulation - more compact - of the master program can be proposed and directly solved without using column generation procedure. For general graph, the auxiliary problem is NP-hard. In this case, an alternative approach is to select some stable set constraints which define a stable set problem in a perfect sub-graph and, to decompose only on these selected constraints. In this way, the master program can be solved in polynomial time where as it is a strengthened formulation of the classical continuous relaxation. The selection of a sub-set of constraints may be difficult but, for mathematical program associated with some particular application, the structure induced by the application itself may be a sufficient guide.

In section 2, we recall the Dantzig-Wolfe decomposition scheme in 0-1 linear programming. Then, in section 3, we present the considered problem namely, the stable set problem with additional linear constraints. In section 4 , we study the gap between the classical continuous relaxation and the linear relaxation of the master problem, obtained by decomposing on stable set constraints, with regards to various stable set polytope representations for general and perfect graphs. For general graphs, an alternative approach 
based on the decomposition on some sub-sets of the stable set constraints is briefly introduced.

\section{The Dantzig-Wolfe decomposition in 0-1 linear program- ming}

Consider the $0-1$ linear program $P$ of the form

$$
(P) \begin{cases}\max c x & \\ A x \leq a & m_{1} \text { constraints } \\ B x \leq b & m_{2} \text { constraints } \\ x \in\{0,1\}^{n} & \end{cases}
$$

where $A \in \mathbb{N}^{m_{1} \times n}$ and $B \in \mathbb{N}^{m_{2} \times n}$ are integer matrices, $c \in \mathbb{N}^{n}, a \in \mathbb{N}^{m_{1}}$ and $b \in \mathbb{N}^{m_{2}}$ are integer vectors. The set $X_{I}^{B}=X^{B} \cap\{0,1\}^{n}$, with $X^{B}=\left\{x \in \mathbb{R}_{+}^{n}: B x \leq b, x \leq 1\right\}$, contains a finite set of vectors, denoted $\left\{x^{1}, x^{2}, \ldots, x^{q}\right\}$, and any point $x \in X_{I}^{B}$ can be represented as

$$
x=\sum_{k=1}^{q} x^{k} \lambda_{k}
$$

with $\sum_{k=1}^{q} \lambda_{k}=1$ and $\lambda_{k} \in\{0,1\} \forall k=1, \ldots, q$.

The main idea of the Dantzig-Wolfe decomposition in integer programming [2] is to rewrite $P$ using the fact that any solution of $P$ is necessarily a solution of $X_{I}^{B}$, as follows:

$$
(M)\left\{\begin{array}{l}
\max \sum_{k=1}^{q} c x^{k} \lambda_{k} \\
\sum_{k=1}^{q} A x^{k} \lambda_{k} \leq a \\
\sum_{k=1}^{q} \lambda_{k}=1 \\
\lambda_{k} \in\{0,1\} \forall k=1, \ldots, q
\end{array}\right.
$$

$M$ is called the master problem.

If we now consider the linear relaxations of $P$ and $M$, denoted $\bar{P}$ and $\bar{M}$ respectively, it is well known that (see for example [2]):

$$
v(\bar{P}) \geq v(\bar{M}) \geq v(M)=v(P)
$$


Indeed, it is easy to see that

$$
(\bar{M})\left\{\begin{array}{l}
\max \sum_{k=1}^{q} c x^{k} \lambda_{k} \\
\sum_{k=1}^{q} A x^{k} \lambda_{k} \leq a \\
\sum_{k=1}^{q} \lambda_{k}=1 \\
\lambda_{k} \geq 0 \forall k=1, \ldots, q
\end{array}\right.
$$

is equivalent to

$$
\left\{\begin{array}{l}
\max c x \\
A x \leq a \\
x \in \operatorname{conv}\left(X_{I}^{B}\right)
\end{array}\right.
$$

where $\operatorname{conv}\left(X_{I}^{B}\right)$ denotes the convex hull of $X_{I}^{B}$. Thus, $v(\bar{P}) \geq v(\bar{M})$ since $v(\bar{P})=$ $\left\{\max c x: A x \leq a, x \in X^{B}\right\}$ and $\operatorname{conv}\left(X_{I}^{B}\right) \subseteq X^{B}$.

Consequently, when the polyhedron $X^{B}$ presents the integrality property, that is to say $X^{B}=\operatorname{conv}\left(X_{I}^{B}\right)$, we have: $v(\bar{P})=v(\bar{M})$. Moreover, when $\operatorname{conv}(X)=\{A x \leq$ a) $\cap \operatorname{conv}\left(X_{I}^{B}\right)$, with $X$ is the set of all $P$ feasible solutions, we have: $v(P)=v(\bar{M})$.

These two previous cases are particular and, in general, we have:

$$
v(\bar{P})>v(\bar{M})>v(M)=v(P)
$$

These inequalities are very important since we need to compute tight upper bound either for exactly solving large-scale linear integer program in a branch and bound scheme, or for evaluating the quality of approximate solutions. In this context, the $M$ formulation and the computation of $v(\bar{M})$ can be really interesting.

Since the number of variables in $\bar{M}$ can be huge (because it is equal to the cardinality of $X_{I}^{B}$ ), a column generation procedure must be applied to compute $v(\bar{M})$. It consists in generating only a subset of variables: those which may participate to an optimal solution. Starting from a subset $J$ of some initial variables (completed with some artificial variables if necessary), we have to solve at each iteration of the column generation procedure the following linear problem:

$$
\left(\bar{M}_{J}\right)\left\{\begin{array}{l}
\max \sum_{k \in J} c x^{k} \lambda_{k} \\
\sum_{k \in J} A x^{k} \lambda_{k} \leq a \\
\sum_{k \in J} \lambda_{k}=1 \\
\lambda_{k} \geq 0 \forall k \in J
\end{array}\right.
$$

and to determine among all remaining variables the one with the greatest reduced cost. The reduced cost of a variable $k$ is defined as follows:

$$
\bar{c}_{k}=c_{k}-\pi A x_{k}-\pi_{0}
$$


with $\pi$ denotes the dual variables associated with constraints $(1)$ and $\pi_{0}$ the dual variable associated with constraint (2). The variable with the greatest strictly positive reduced cost (or several variables with strictly positive reduced cost - see [2] for details concerning column generation procedures) must be included in $J$ and $\bar{M}_{J}$ must be reoptimized. The procedure stops when all remaining variables have negative or null reduced cost, and the optimal solution of $\bar{M}_{J}$ is thus an optimal solution of $\bar{M}$.

The problem of determining a variable, or equivalently a solution of $X_{I}^{B}$, with the greatest reduced cost, called the auxiliary problem denoted Aux, can be formulated as follows:

$$
(A u x)\left\{\begin{array}{l}
\max (c-\pi A) x-\pi_{0} \\
B x \leq b \\
x \in\{0,1\}^{n}
\end{array}\right.
$$

In the following, we focus on a particular class of $\operatorname{conv}\left(X_{I}^{B}\right)$ polyhedron: the stable set polytope.

\section{The considered problem: the stable set problem with additional linear constraints}

In this paper, we suppose that the $m_{2}$ constraints $B x \leq b$ are of the form

$$
\sum_{i \in Q_{k}} x_{i} \leq 1 \text { with } Q_{k} \subseteq\{1, \ldots, n\} \text { and }\left|Q_{k}\right| \geq 2 \forall k=1, \ldots, m_{2} .
$$

These constraints can also be represented by a graph $G=(V, E)$ defined as follows:

- each variable $x_{i}, i=1, \ldots, n$, is represented by a vertex $i \in V$ (thus $|V|=n$ ),

- each constraint $k, k=1, \ldots, m_{2}$, is represented by one or several edges $(i j)$ of $E$ for all $i$ and $j$ (with $i<j$ ) belonging to $Q_{k}$.

A solution $x$ belonging to $X_{I}^{B}$ can be seen as the incidence vector of a set $S \subseteq V$ of vertices (with $x_{i}=1$ if $i \in S$ and 0 otherwise) such that: $\forall(i, j) \in S \times S,(i j) \notin E$. Consequently, $S$ is a stable set in $G$ and $\operatorname{conv}\left(X_{I}^{B}\right)$ is exactly the stable set polytope, usually denoted by $\operatorname{STAB}(G)$ :

$$
\operatorname{STAB}(G)=\operatorname{conv}\left(x^{S} \in\{0,1\}^{|V|}: S \subseteq V \text { is a stable set in } G\right) .
$$


In this context, the considered program $P$ corresponds to the maximum weighted stable set problem with additional linear constraints (the first $m_{1}$ constraints).

The stable set polytope and its LP-relaxation has been the subject of numerous studies (see i.e [8]).

Considering an arbitrary graph $G=(V, E)$, the edge constraints of the form

$$
x_{i}+x_{j} \leq 1 \forall(i j) \in E
$$

are facet-defining inequalities of $\operatorname{STAB}(G)$. And we have:

$$
\operatorname{STAB}(G)=\operatorname{conv}\left(x \in\{0,1\}^{|V|}: x \text { satisfies all edge constraints }\right)
$$

In general cases, the edge polytope defined as follows

$$
\operatorname{ESTAB}(G)=\left\{x \in \mathbb{R}_{+}^{|V|}: x \leq 1 \text { and satisfies all edge constraints }\right\}
$$

is not equal to the stable set polytope (except for bipartite graphs, see i.e [8]). In other words, all edge constraints are not sufficient to describe the stable set polytope, additional facet-defining inequalities must be introduced.

Obviously, maximal clique constraints of that form

$$
\sum_{i \in Q} x_{i} \leq 1, \text { with } Q \subseteq V
$$

where $Q$ is a maximal clique in $G$, generalize edge constraints. The clique polytope (also called fractional stable set polytope) is defined as follows

$$
\operatorname{QSTAB}(G)=\left\{x \in \mathbb{R}_{+}^{|V|}: x \text { satisfies all maximal clique constraints }\right\}
$$

For arbitrary graph, we have $\operatorname{STAB}(G) \subseteq \mathrm{QSTAB}(G) \subseteq \operatorname{ESTAB}(G)$ (see i.e. [8]). But, if $G$ is a perfect graph, it appears that $\operatorname{STAB}(G)=\operatorname{QSTAB}(G)$.

In the next section, we apply the Dantzig-Wolfe decomposition scheme on stable set constraints and we compare linear relaxations of master and initial programs with regards to various stable set polytope representations. 


\section{Dantzig-Wolfe decomposition on stable set constraints: a way to strengthen the LP-relaxation}

\subsection{Gap between LP-relaxations of master and initial problems}

In section 2, we recall that the LP-relaxation of the master problem in a Dantzig-Wolfe decomposition scheme is equivalent to

$$
\left\{\begin{array}{l}
\max c x \\
A x \leq a \\
x \in \operatorname{conv}\left(X_{I}^{B}\right)
\end{array}\right.
$$

When $\operatorname{conv}\left(X_{I}^{B}\right)$ exactly corresponds to a stable set polytope, $\bar{M}$ can be reformulated as follows:

$$
\left\{\begin{array}{l}
\max c x \\
A x \leq a \\
x \in \operatorname{STAB}(G)
\end{array}\right.
$$

Consequently, if $G$ is an arbitrary graph, $v(\bar{M}) \leq v(\bar{P})$ since $\operatorname{STAB}(G) \subseteq X^{B}$. Otherwise, if $G$ is a perfect graph or the complementary graph of a perfect graph, $\operatorname{STAB}(G)$ can be replaced by $\operatorname{QSTAB}(G)$ (since in this case $\operatorname{STAB}(G)=\mathrm{QSTAB}(G)$ ) and we have:

- if $\operatorname{QSTAB}(G) \subset X^{B}, v(\bar{M}) \leq v(\bar{P})$,

- if $\operatorname{QSTAB}(G)=X^{B}, v(\bar{M})=v(\bar{P})$.

Consequently, when $X^{B}$ does not exactly describe the clique polytope, the LP-relaxation of the master problem in a Dantzig-Wolfe decomposition scheme may give a tighter upper bound than the LP-relaxation of the initial problem. The gap comes from the difference between the two polytopes $X^{B}$ and $\operatorname{QSTAB}(G)$.

Moreover, the auxiliairy problem $A u x$ can be reformulated as follows:

$$
\left\{\begin{array}{l}
\max (c-\pi A) x-\pi_{0} \\
x \in \operatorname{STAB}(G)
\end{array}\right.
$$

Thus, $A u x$ is the maximal weight stable set problem. If $G$ is an arbitrary graph, the problem is NP-hard ; otherwise, if $G$ is a perfect graph, this problem can be solved in polynomial time. 
In this context, when $\operatorname{STAB}(G) \subset X^{B}$, an obvious way for strengthening $\bar{P}$ is to add in $X^{B}$ some additional facet-defining inequalities for $\operatorname{STAB}(G)$ (this is done by a solver like Cplex when it determines the so-called clique cut - cf. [4]). When $G$ is a perfect graph, replacing $X^{B}$ by $\operatorname{QSTAB}(G)$ remains to compute $v(\bar{M})$. In this case, the difficulty comes from the possible huge number of maximal clique constraints. Indeed, excepted for some particular graphs (like interval graph for example) for which the number of maximal cliques is bounded $(O(n)$ for interval graph, see for example [7]), there exists perfect graphs with exponentially many maximal cliques. However, this problem can be avoided using either constraints generation techniques or, if possible, a more compact reformulation. In the following section, we focus on a particular class of graphs, namely cocomparability graphs, and we show how to obtain a more compact formulation for $\bar{M}$.

\subsection{The case of cocomparability graph}

A simple graph is a comparability graph if it has a transitive orientation, i.e., an orientation of edges such that $(i, j)$ and $(j, k)$ implies $(i, k)$. In [7,9], polynomial time algorithms are proposed for determining if a graph is a comparability graph, and for such graphs, for defining a transitive orientation in polynomial time. A cocomparability graph is the complement of a comparability graph. Cocomparability graph is perfect graph but it can have an exponential number of maximal cliques. However it exists a more compact formulation of the stable set polytope using flow variables (as also suggested in [1]).

Let us consider $G=(X, U)$ a cocomparability graph. A stable set in $G$ corresponds to a clique in its complement graph, denoted $G_{C}=\left(X, U_{C}\right)$ with $\left|U_{C}\right|=m$. Since $G_{C}$ is a comparability graph, we can define $\overrightarrow{G_{C}}=\left(X, \overrightarrow{U_{C}}\right)$ a digraph obtained by choosing a transitive orientation of $U_{C}$. Let us add into $\overrightarrow{G_{C}}$ two fictitious vertices $e$ and $s$ and the following $\operatorname{arcs}$ in $\overrightarrow{U_{C}}:(e, s),(e, i)$ and $(i, s)$ for all $i=1, \ldots, n$. Thus, any stable set in $G$ define a path between $e$ and $s$ in $\overrightarrow{G_{C}}$ and conversely. Consequently, it is possible to represent $\operatorname{STAB}(G)$, or equivalently $\operatorname{QSTAB}(G)$, by a classical vertex-arc formulation of paths.

In the vertex-arc formulation of the stable set polytope, each variable $\phi_{j k}$ represents an $\operatorname{arc}(j, k)$ in $\overrightarrow{U_{C}}$, and $\phi=\left(\phi_{j k}\right)_{(j, k) \in \overrightarrow{U_{C}}}$ is an incidence vector of a path between $e$ and $s$ in $\overrightarrow{G_{C}}$ if it satisfies the following constraints :

$$
\left\{\begin{array}{l}
\sum_{k \in \Gamma^{+}(j)} \phi_{j k}-\sum_{k \in \Gamma^{-}(j)} \phi_{k j}=0 \quad \forall j=\{1, \ldots, n\} \\
\sum_{k \in \Gamma^{+}(e)} \phi_{e k}=1 \\
-\sum_{k \in \Gamma^{-}(s)} \phi_{k s}=-1 \\
\phi \in\{0,1\}^{m}
\end{array}\right.
$$


The number of variables is equal to the number of arcs in $\overrightarrow{U_{C}}$, i.e. $m+2 n+1$ or equivalently $O\left(n^{2}\right)$ ), while the number of contraints equals $n+2+m_{1}$ or equivalently $O\left(n^{2}\right)$. Moreover, it is well-known that this polyhedron presents the integrality property (since the constraint matrix is a vertex-arcs incidence matrix).

As we show in the previous section, a way to strengthened $\bar{P}$ (and for perfect graph to reach the bound provided by the Dantzig-Wolfe decomposition scheme) is to replace $X^{B}$ by $\operatorname{QSTAB}(G)$. For cocomparability graph, a way to describe $\operatorname{QSTAB}(G)$ with a polynomial number of variables and constraints is to use the vertex-arc formulation as follows:

$$
\begin{cases}\max \sum_{j=1}^{n} c_{j} \sum_{k \in \Gamma^{+}(j)} \phi_{j k} & \\ \sum_{j=1}^{n} a_{i j} \sum_{k \in \Gamma^{+}(j)} \phi_{j k} \leq a_{i} & \forall i=1, \ldots, m_{1} \\ \sum_{k \in \Gamma^{+}(j)} \phi_{j k}-\sum_{k \in \Gamma^{-}(j)} \phi_{k j}=0 & \forall j=\{1, \ldots, n\} \\ \sum_{k \in \Gamma^{+}(e)} \phi_{e k}=1 & \\ -\sum_{k \in \Gamma^{-}(s)} \phi_{k s}=-1 & \\ 0 \leq \phi_{j k} \leq 1 \forall(j, k) \in \overrightarrow{U_{C}} & \end{cases}
$$

where $x$ and $\phi$ variables are linked that way:

$$
x_{j}=\sum_{k \in \Gamma^{+}(j)} \phi_{j k} \forall j=1, \ldots, n .
$$

Consequently, this new formulation must be used to compute $v(\bar{M})$.

\subsection{A decomposition scheme for general graphs}

For general graph $G$, the LP-relaxation of the master program obtained by applying Dantzig-Wolfe decomposition scheme on all the stable set constraints, cannot be solved in polynomial time because the auxiliary problem is NP-hard. An alternative approach is to identify one or several perfect sub-graphs of $G$ and to decompose only on the constraints describing these subgraphs as follows:

1. determine one or several perfect subgraphs $G^{k}=\left(V^{k}, U^{k}\right)$ with $k=1, \ldots, K$ $(K \geq 1)$ of $G$,

2. separate the constraints set $B x \leq b$ into $K+1$ subsets: for $k=1, \ldots, K, B^{k} x \leq b^{k}$ are all the stable set constraints represented in $G^{k}, \bar{B} x \leq \bar{b}$ are all the remaining constraints,

3. apply Dantzig-Wolfe decomposition on $B^{k} x \leq b^{k}$, with $k=1, \ldots, K$. 
The obtained master program denoted $M^{\prime}$ is equivalent to:

$$
\left\{\begin{array}{l}
\max c x \\
A x \leq a \\
\bar{B} x \leq \bar{b} \\
x \in \operatorname{STAB}\left(G^{k}\right) \quad \forall k=1, \ldots, K
\end{array}\right.
$$

Thus, $v\left(\bar{M}^{\prime}\right)$ is tractable and we have $: v(\bar{P}) \geq v\left(\bar{M}^{\prime}\right) \geq v(\bar{M})$. For computing $v\left(\bar{M}^{\prime}\right)$, several identical methods can be used: a direct computation with column generation procedure, a constraint generation procedure applied to the clique polytope formulation of $\operatorname{STAB}\left(G^{k}\right)$ and, when $\operatorname{QSTAB}\left(G^{k}\right)$ can be described with a "polynomial" number of clique constraints for all $k=1, \ldots, K$, a direct resolution of the clique polytope formulation.

In this approach, the determination of one or several perfect subgraphs may be very difficult for general graph. But, for a graph associated with a real application, the structure of the problem may be a powerful guide for identifying some sub-graphs with "nice" property.

\section{Conclusion}

When solving exactly or approximately large scale integer linear program $(I L P)$, a very important information comes from the computation of the linear relaxation of $I L P$. It is well-known that, when applying Dantzig-Wolfe decomposition on $I L P$, the linear relaxation of the master problem $\bar{M}$ may present a smaller integrality gap. We consider here a particular class of $I L P$, those which contain a sub-set of constraints defining a stable set polytope. In this case, when decomposition is performed on the stable set constraints, the difference between LP-relaxations of $I L P$ and $M$ can be partially explained by the gap between the stable set polytope and its corresponding LP-relaxation. In case of perfect graph, we show that the LP-relaxation of $M$ is equivalent to another formulation obtained by replacing in $I L P$ the inequalities defining the stable set polytope by the constraints defining the clique polytope. A more compact formulation is then given for cocomparability graphs, based on vertex-arc formulation of paths. In case of general graph, $\bar{M}$ cannot be computed in polynomial time. Thus an alternative approach is possible in which stable set constraints are separated in order to determine some subsets with "nice" property. This is the subject of future researchs. 


\section{References}

[1] P. Barcia, J.O. Cerdeira (2003) Node packings on cocomparability graphs, Operations Research Letters 31:341-342

[2] C.Barnhart, E.L.Johnson, G.L.Nemhauser, M.W.P.Savelsbergh, P.H.Vance (1998) Branch-and-Price: Column Generation for Solving Huge Integer Programs, Operations Research 46(3):316-329

[3] E.Bensanna, G.Verfaillie, J-C.Agnèse, N.Bataille, D.Blumstein (1996) Exact and approximate methods for the daily management of an earth observation satellite, Proc. of the $4^{\text {th }}$ Int. Symposium on Space Mission Operations and Ground Data Systems, Munich, Germany

[4] ILOG Cplex 8.0 User's Manual.

[5] G.B. Dantzig, P. Wolfe (1960) Decomposition Principle for Linear programs, Operations Research 8:101-111

[6] V. Gabrel, C. Murat (2003) Mathematical programming for Earth observation satellite mission planning, in Operations Research in Space and Air, T.A. Ciriani, G. Fasano, S. Gliozzi, R. Tadei editors, Kluwer Academics Publishers:103-122

[7] P.C. Gilmore, A.J. Hoffman (1964) A characterization of comparability graphs and of interval graphs, Can. Journal. Math. 16:539-548

[8] M. Grötschel, L.Lovàsz, A. Schrijver (1988) Geometric Algorithms and Combinatorial Optimization, Springer-Verlag

[9] A. Pnueli, A. Lempel, S. Even (1971) Transitive orientation of graphs and identification of permutation graphs, Can. Journal. Math. 23(1):160-175 